\title{
Smart Grids aus der Sicht der Informations- und Kommunikationstechnik
}

S. Bessler

Online publiziert am 23. Mai 2014

(c) Springer Verlag Wien 2014

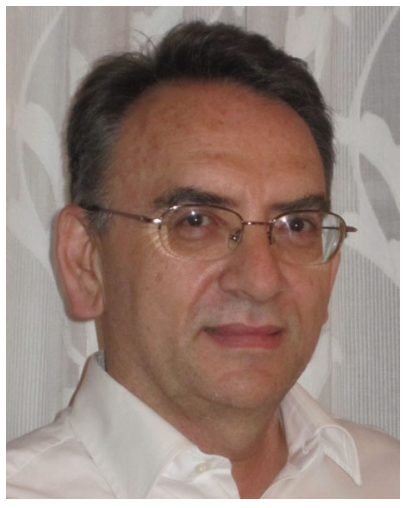

Sandford Bessler
Liebe Leserinnen und liebe Leser,

unsere Elektrizitätsversorgung befindet sich im Wandel. Zum einen steigt der Energiebedarf kontinuierlich an, zum anderen stehen die Energieversorger und Netzbetreiber durch die verstärkte Einbindung von regenerativen Energien, unter anderem gewonnen aus Wind und Photovoltaik, vor großen Herausforderungen. Das oberste Ziel ist dabei, eine nachhaltige Entwicklung der Energieversorgung und -bereitstellung in unserem Land bzw. in Europa zu gewährleisten. Als Lösung für die künftige Energieversorgung werden Smart Grids angesehen. Die Stromversorgung wird sich damit von dem bisherigen zentral gesteuerten zu einem intelligenten, dezentralen System weiter entwickeln. Neben den Herausforderungen für die Energietechnik kommen durch Smart Grids auch auf die Informations- und Kommunikationstechnik (IKT) große Aufgaben zu, die es zu lösen gilt.

Bereits in e\&i-Heft 4/5 2013 war der Themenschwerpunkt der IKT zur Realisierung der „intelligenten Netze” gewidmet. Mit der aktuell vorliegenden Ausgabe wollen wir die Berichterstattung über Entwicklungen in diesem Bereich fortsetzen. Sechs Beiträge, die sich mit der IKT-Seite von Smart Grids-Projekten in Österreich befassen, wurden dafür ausgewählt:

Zunächst führt ein Bericht der Autoren Hermann Bühler, Thomas Györgyfalvay und Roman Zelenka zur Thematik der Smart Grids hin und beleuchtet vor allem das Vorgehen, die Konzeption und die Modellierung von "smarten" Kommunikationsnetzen.
Im darauf folgenden Bericht erläutern Helmut Leopold und Thomas Bleier mit Projektbeispielen die Bedeutung der Sicherheit im Migrationspfad der Elektrizitätsnetze zu Smart Grids und gehen weiters auf die Innovationsdynamik durch die intelligenten Netze ein.

Kurt Leonhartsberger und Momir Tabakovic geben einen Überblick über österreichische Smart Grid-Forschungsaktivitäten im nationalen und internationalen Kontext und zeigen konkrete Beispiele anhand der österreichischen Modellregionen Oberösterreich, Salzburg sowie den Biosphärenpark Großes Walsertal und die dort entwickelten smarten Lösungsansätze.

Der Beitrag von Tobias Deutsch, Friedrich Kupzog und Alfred Einfalt mit dem Titel "Integration von Energiemarkt und Verteilnetzbetrieb durch einen Flexibility Operator" beschreibt, wie Flexibilitätsinformation im Energieverbrauch der Kunden für die kapazitätsund marktbedingte Steuerung der Energieströme verwendet werden kann.

Der Beitrag von Markus Schwarz und Andrea Kollmann widmet sich der wirtschaftlichen Betrachtung von Netzausbau und Smart Grid-Lösungen in der Niederspannungsebene und zeigt verschiedene Möglichkeiten für die Integration von PV-Strom in bestehenden Niederspannungsnetzen.

Das Thema Smart Grid ist eng mit der Qualität der Kommunikation und mit der Netzsicherheit verbunden. In ihrem Beitrag "Security Challenges for Wide Area Monitoring in Smart Grids" geben Tanja Zseby und Joachim Fabini einen Überblick über Weitverkehrsnetze für das Monitoring von Smart Grids: Es werden Szenarien, Protokolle und insbesondere Mechanismen zur Erhaltung der Netzsicherheit erläutert.

Damit wünschen wir Ihnen eine informative Lektüre der Beiträge dieses e\&i-Themenschwerpunktes und freuen uns auf Ihre Rückmeldungen.
Bessler, Sandford, FTW Forschungszentrum Telekommunikation Wien GmbH Donau-City-Straße 1, 1220 Wien, Österreich (E-Mail: bessler@ftw.at) 